\title{
Mineral Mapping with Hyperspectral Data A Case Study over the Moses Rock Dyke and Mule Ear Diatreme (UTAH, USA)
}

\author{
V. Zichella ${ }^{1}$, A. A. de Gasparis ${ }^{1}$ and N. E. Pendock ${ }^{2}$
}

1. Geophysical Services Department of Anglo American Corporation, 45 Main Street, Johannesburg 2000

2. Department of Computational and Applied Mathematics, University of the Witwatersrand, PO Box 197, WITS 2050 (under contract to Anglo American`s Geophysical Services Department)

Two AVIRIS scenes acquired in 1995 over the Moses Rock Dyke - Mule Ear area in southeastern Utah, USA, were processed for mineral mapping purposes (Fig. 1).

The area investigated is characterised by the presence of Tertiary ultramafic intrusions within a Late Carboniferous to Jurassic sedimentary succession. The ultramafic intrusion represented an excellent target for the fine-tuning of the investigation methods towards the identification of kimberlites, due to their similar groundmass mineralogy and weathering products. Abundant carbonates and shales within the host rocks created other interesting targets for the investigation of spectral properties of rocks and minerals with airborne hyperspectral data (Fig. 2).

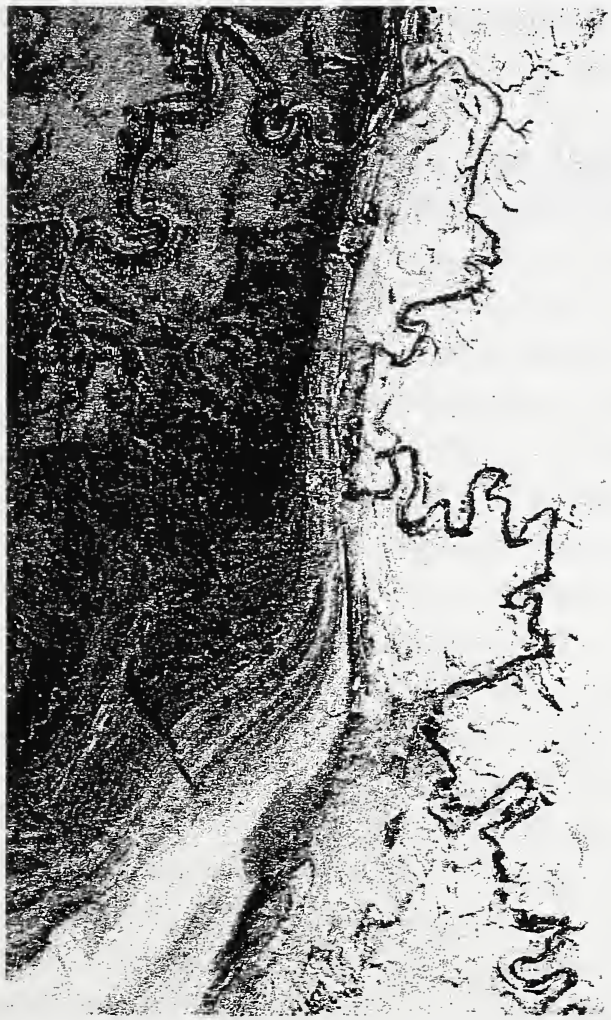

Fig. 1. AVIRIS scenes 950724B09-2/3. Bands 20-100-200.

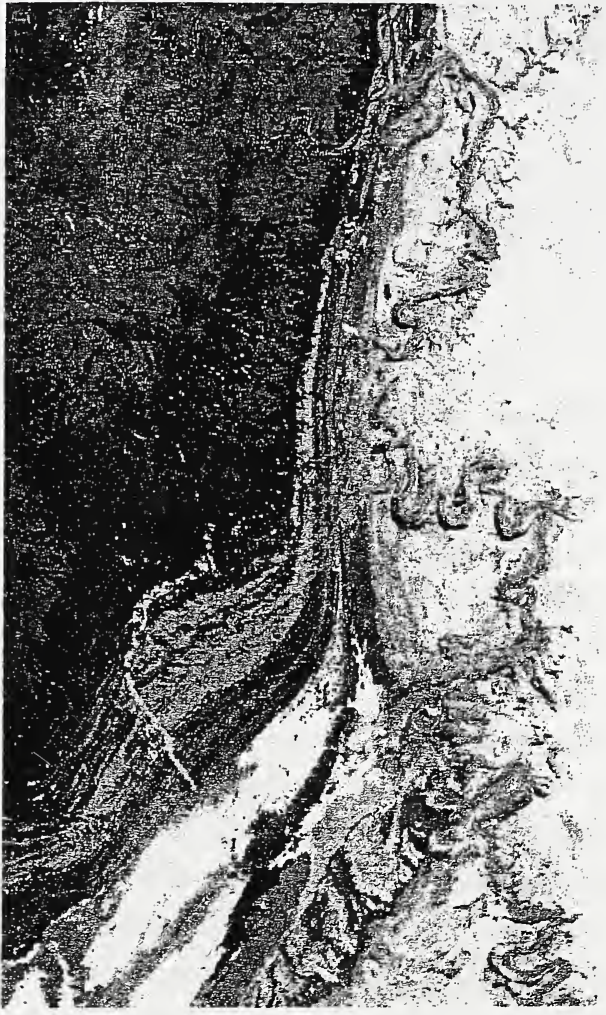

Fig. 2. Mineral map of the Moses Rock / Mule Ear area. 
Mapping lithologies in a multivariate remotely sensed image presupposes that individual lithologies may be separated spectrally. If we assume this to be so we have two very powerful pieces of prior information:

- individual geological units of interest will be relatively rare in a whole image

- lithological responses are often spatially concentrated and some guess could be made at approximate minimum and maximum sizes. Shape information could also be used in some applications.

Classical statistics is very good at describing the average behaviour of a multivariate data set. Our first piece of prior information tells us to look for outliers which may be either noise or targets and so the usual statistical approaches of principal components analysis or classification are of limited use. Noise in hyperspectral imagery comes from many sources including atmospheric effects, aircraft motion and sensor noise. Searching for outliers will thus produce many candidate spectra that may then be winnowed using our second prior assumption of spatial contiguity and morphology.

Atmospheric correction and spectral mixture modelling techniques were developed and tested in the course of this study and the results obtained were very encouraging.

Fast, automatic techniques combined with a critical interpretation of the results provided the key to the isolation and identification of anomalous (spatially limited) mineral associations that can become targets for exploration.

Comparison with state of the art public domain software showed the advantages of the in-house software in the identification of diluted or small spectral features in complex and noisy data. 